\title{
Monte Carlo calculations of few-body and light nuclei
}

\author{
R. B. Wiringa
}

JAN 31

Physics Division, Argonne National Laboratory, Argonne, Illinois 6C439, USA

\begin{abstract}
A major goal in nuclear physics is to understand how nuclear structure comes about from the underlying interactions between nucleons. This requires modelling nuclei as collections of strongly interacting particles. Using realistic nucleon-nucleon potentials, supplemented with consistent three-nucleon potentials and two-body electroweak current operators, variational Monte Carlo methods are used to calculate nuclear ground-state properties, such as the binding energy, electromagnetic form factors, and momentum distributions. Other properties such as excited states and low-energy reactions are also calculable with these methods.
\end{abstract}

\section{HAMILTONIAN}

Quantum chromodynamics gives some hope of eventually understanding the residual strong force between nucleons. However, it has not yet been developed to the point where it can make quantitative predictions for nucleon-nucleon (NN) interactions. For studying nuclear many-body systems we generally must rely on potential representations, where the choice of potential forms is guided by meson-exchange theory. To fit NN scattering data and deuteron properties requires a complicated operator structure for the NN potential. Further, mesonexchange theory and the existence of low-energy nucleon resonances suggest that there should be significant many-body forces, which require three-nucleon (NNN) or higher-order potentials in a nucleons-only representation. In practice, NN potentials give the major contribution to the energy and induce the major correlations in the nuclear wave function, but NNN potentials are needed for quantitative agreement with nuclear masses and can contribute a significant fraction of the total binding.

A realistic nuclear Hamiltonian can be written in the form:

$\mathrm{H}=\frac{-\hbar^{2}}{2 \mathrm{~m}} \sum_{\mathrm{i}} \nabla_{\mathrm{i}}^{2}+\sum_{\mathrm{i}<\mathrm{j}} \mathrm{v}_{\mathrm{ij}}+\sum_{\mathrm{i}<\mathrm{j}<\mathrm{k}} \mathrm{V}_{\mathrm{ijk}}$,

where $\mathrm{v}_{\mathrm{ij}}$ is an NN potential fit to elastic scattering data and deuteron properties, and $\mathrm{V}_{\mathrm{ijk}}$ is an NNN potential fit to many-body ground state energies. Most realistic NN potentials can be written in an operator form:

$v_{i j}=\sum_{p=1, n} v_{p}\left(r_{i j}\right) O_{i j}^{p}$,

where the first fourteen operators are: 


$$
\begin{aligned}
O_{i j}^{p}= & 1, \tau_{i} \cdot \tau_{j}, \sigma_{i} \cdot \sigma_{j}, \quad\left(\sigma_{i} \cdot \sigma_{j}\right)\left(\tau_{i} \cdot \tau_{j}\right), S_{i j}, S_{i j}\left(\tau_{i} \cdot \tau_{j}\right), L \cdot S, L \cdot S\left(\tau_{i} \cdot \tau_{j}\right), \\
& L^{2}, L^{2}\left(\tau_{i} \cdot \tau_{j}\right), L^{2}\left(\sigma_{i} \cdot \sigma_{j}\right), L^{2}\left(\sigma_{i} \cdot \sigma_{j}\right)\left(\tau_{i} \cdot \tau_{j}\right),(L \cdot S)^{2},(L \cdot S)^{2}\left(\tau_{i} \cdot \tau_{j}\right) .
\end{aligned}
$$

The first eight operators are required phenomenologically to reproduce S- and P-wave NN scattering and deuteron properties. The additional L-dependent operators are required for fitting higher partial waves. Examples are the Reid v8 potential [1], an adaptation of the standard Reid soft-core potential which uses the first eight operators above, and the Argonne $v_{14}$ potential [2], which uses all fourteen operators shown. Other realistic potentials, such as the Bonn [3], Nijmegen [4], and Paris [5] models, use $\mathrm{p}^{2}$ operators instead of, or in addition to, $\mathrm{L}^{2}$ operators.

The meson-theoretic basis for the NN potential is illustrated in Figure 1. The dominant long-range interaction is one-pion exchange, which has the operator structure:

$$
v_{i j}^{\pi}=X_{i j}^{\pi}\left(\tau_{i} \cdot \tau_{j}\right)=\left[Y\left(r_{i j}\right) \sigma_{i} \cdot \sigma_{j}+T\left(r_{i j}\right) S_{i j}\right] \tau_{i} \cdot \tau_{j},
$$

where $Y(r)$ and $T(r)$ are the usual Yukawa and tensor functions. At intermediate ranges, twopion exchange, with the possible excitation of intermediate $\Delta$-isobar resonances, is the dominant reaction. Analysis with interaction models that include explicit $\Delta$ degrees of freedom [2] show that these processes provide significant attraction, which is predominantly central in character, but includes important components for the first six ciperators of eq.(3). At shorter ranges the interaction may be dominated by the exchange of heavier mesons, such as the $\rho$ and $\omega$, or the quark substructure of nucleons may start to play some more complicated role; these features contribute to the spin-orbit and higher-order terms in the potential.

Realistic models for the NNN potential also have a non-trivial operator dependence. Meson-exchange diagrams contributing to the NNN potential are shown in Figure 2, starting with a long-range two-pion-exchange part of the Fujita-Miyazawa form [6]:

$V_{i j k}^{2 \pi}=A \sum_{c y c}\left(\left\{X_{i j}^{\pi}, X_{i k}^{\pi}\right\}\left\{\tau_{i} \cdot \tau_{j}, \tau_{i} \cdot \tau_{k}\right\}+\frac{1}{4}\left[X_{i j}^{\pi}, X_{i k}^{\pi}\right]\left[\tau_{i} \cdot \tau_{j}, \tau_{i} \cdot \tau_{k}\right]\right)$,

which is built up from the one-pion-exchange operators, and arises from an intermediate $\Delta$ isobar excitation. In the Urbana NNN potentials [7] a phenomenological intermediate-range repulsive term is added:

$V_{i j k}^{R}=U \sum_{c y c} T^{2}\left(r_{i j}\right) T^{2}\left(r_{j k}\right)$

which can be viewed as an interference of the intermediate-range attraction between two NN

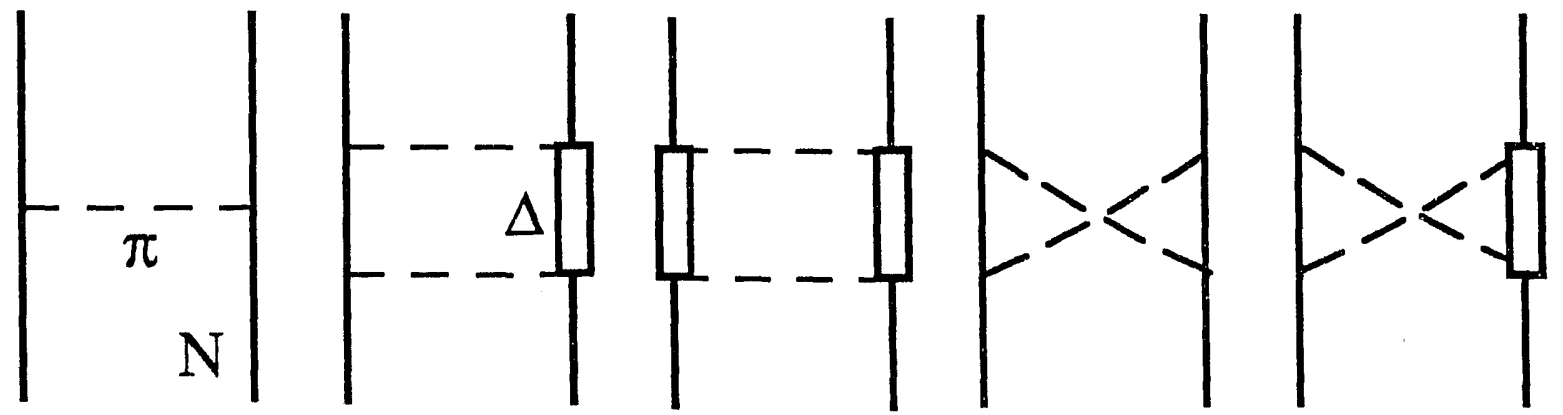

Figure 1. Diagrams contributing to the NN potential. 
pairs sharing one nucleon. The practical effect of adding such an NNN potential is additional binding in light nuclei where the attractive long-range two-pion-exchange dominates, and more repulsion in nuclear matter where the intermediate-range repulsive term produces a more rapid saturation. The strengths of the attractive and repulsive parts, $A$ and $U$, are adjusted to give an overall best fit to nuclear binding energies in these two regimes.

Below we report results for two models: Urbana VII, which was fit to earlier variational calculations of light nuclei and nuclear matter [8] and is used here for closed-shell nuclei, and Urbana VIII, which was fit to more recent exact Faddeev and Green's function Monte Carlo (GFMC) calculations and is used here for few-body nuclei [9]. Other NNN potentials are available, such as the Tucson-Melbourne model [10], which has a more complete two-pionexchange term.

\section{VARIATIONAL TRIAL FUNCTIONS}

The variational method can be used to obtain approximate solutions of the many-body Schrödinger equation for Hamiltonians of the kind given above and for a wide range of nuclear systems: few-body nuclei [7-9] such as $3 \mathrm{H}$ and ${ }^{4} \mathrm{He}$, light nuclei [11] such as $16 \mathrm{O}$ and ${ }^{40} \mathrm{Ca}$, nuclear matter $[12,13]$ and neutron stars [14]. A suitably parametrized trial function $\Psi_{v}$ is used to calculate an upper tound $E_{\mathrm{v}}$ to the ground-state energy $E_{0}$ using the Rayleigh-Ritz variational principle:

$E_{v}=\frac{\left\langle\Psi_{v}|H| \Psi_{v}\right\rangle}{\left\langle\Psi_{v} \mid \Psi_{v}\right\rangle} \geq E_{0}$

The parameters in $\Psi_{\mathrm{V}}$ are varied to minimize $\mathrm{E}_{\mathrm{V}}$, and the lowest value is taken as the approximate ground-state energy. 'The corresponding $\Psi_{\mathrm{v}}$ can then be used to calculate other properties of interest. A better energy may be obtained by using $\Psi_{v}$ as a starting point for a perturbation or GFMC calculation [15]. The key steps are always to 1) find a good ansatz for $\Psi_{\mathrm{v}}$, and 2 ) accurately evaluate the expectation value $\langle\mathrm{H}\rangle$.

The strong state-dependence of the interactions induces corresponding correlations in the wave function. A good trial function can be constructed from a product of correlation operators:

$\left|\Psi_{\mathrm{v}}\right\rangle=\left[1+\sum_{\mathrm{i}<\mathrm{j}} \mathrm{U}_{\mathrm{ij}}^{\mathrm{LS}}+\sum_{\mathrm{i}<\mathrm{j}\langle\mathrm{k}} \mathrm{U}_{\mathrm{ijk}}^{\mathrm{TN}}\right]\left[S \prod_{\mathrm{i}<\mathrm{j}}\left(1+\mathrm{U}_{\mathrm{ij}}\right)\right]\left|\Psi_{\mathrm{J}}\right\rangle$,

where $\Psi_{\mathrm{J}}$ is a Jastrow wave function, and $\mathrm{U}_{\mathrm{ij}}, \mathrm{U}_{\mathrm{ij}}^{\mathrm{LS}}$, and $\mathrm{U}_{\mathrm{ijk}}^{\mathrm{TNI}}$ are two- and three-body

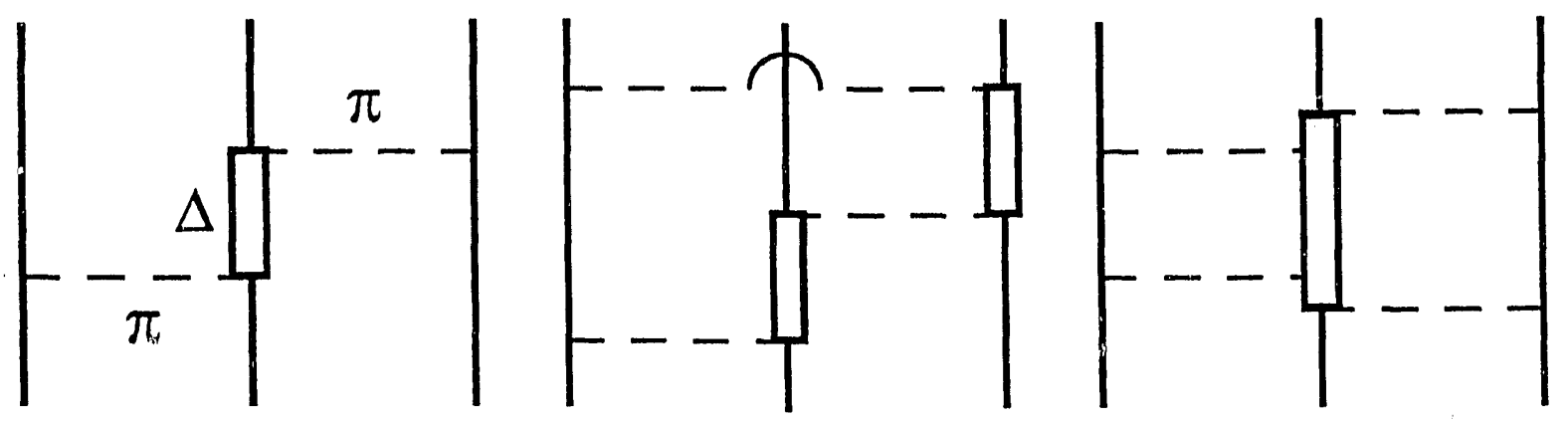

Figure 2. Diagrams contributing to the NNN potential. 
correlation operators:

$$
\begin{aligned}
& \left|\Psi_{J}\right\rangle=\prod_{i<j} f_{c}\left(r_{i j}\right)\left|\Phi_{A}\left(J M T_{3}\right)\right\rangle, \\
& U_{i j}=\sum_{p=2,6}\left[\prod_{k \neq i, j} f_{3}\left(r_{i j}, r_{j k}, r_{k i}\right)\right] u_{p}\left(r_{i j}\right) O_{i j}^{p}, \\
& U_{i j}^{L S}=\sum_{p=7,8}\left[\prod_{k \neq i, j} f_{3}\left(r_{i j}, r_{j k}, r_{k i}\right)\right] u_{p}\left(r_{i j}\right) O_{i j}^{p}, \\
& U_{i j k}^{T N I}=\varepsilon V_{i j k}\left(\tilde{r}_{i j}, \tilde{r}_{j k}, \tilde{r}_{k i}\right) .
\end{aligned}
$$

The operators $O_{i j}^{p}$ are the same as in eq.(3); since they are non-commuting, the symmetrization operator $S$ is required in the product of eq.(8). The first six operators have a nice closed algebra and are conveniently treated together in eq.(10). The spin-orbit (LS) correlations of eq.(11) are more expensive to compute because of their gradient operators and only a linear term is used in eq.(8), while no correlations have been introduced for $\mathrm{L}^{2}$ or higher terms in the interaction. The $f_{3}\left(r_{i j}, r_{j k}, r_{k i}\right)$ in eqs. (10)-(11) is a supplemental three-body correlation that reduces the noncentral correlation when a third particle comes between the correlated pair. The three-nucleon interaction (TNI) correlation of eq.(12) includes all the operator dependence of eq. (5); $\varepsilon$ is a small negative number and $\widetilde{\mathrm{r}}$ a scaled radial variable.

The $\Phi_{A}$ is an antisymmetric single-particle state, with appropriate properties for the system of interest, e.g., the quantum numbers $\mathrm{JMTT}_{3}$ for a particular nucleus. For few-body nuclei we have used a single-particle $\Phi_{\mathrm{A}}$ with spin-isospin indices and no spatial dependence, e.g., for ${ }^{4} \mathrm{He}:$

$\left|\Phi_{4}\left(\mathrm{MMTT}_{3}\right)\right\rangle=\left|\Phi_{\alpha}(0000)\right\rangle=A|\mathrm{p} \uparrow \mathrm{p} \ln \uparrow \mathrm{n} \downarrow\rangle$

For closec-shell nuclei like $16 \mathrm{O}$ and ${ }^{40} \mathrm{Ca}$ we use a product of four jeterminants, one for spinup protons, one for spin-down protons, etc.:

$\left|\Phi_{16}\left(\mathrm{JMTT}_{3}\right)\right\rangle=\left|\Phi_{\mathrm{O}}(0000)\right\rangle=\Sigma(-1)^{\mathrm{P}} \mathrm{D} \uparrow_{\mathrm{p}} \mathrm{D} \downarrow_{\mathrm{p}} \mathrm{D} \uparrow_{\mathrm{n}} \mathrm{D} \downarrow_{\mathrm{n}}$

For 160 each determinant is constructed from one $1 \mathrm{~s}$ and three $1 \mathrm{p}$ radial functions. A term in the sum (14) is obtained by letting particles $i_{1}$ to $i_{4}$ be $\uparrow p$, is to $i_{8}$ be $\downarrow p$, is to $i_{12}$ be $\uparrow n$, and $i_{13}$ to $i_{16}$ be $\downarrow$ n. The sum is over all partitions of the 16 nucleons into four groups of four particles, and the sign $(-1)^{\mathrm{P}}$ is chosen so that $|\Phi\rangle$ is antisymmetric. For ${ }^{40} \mathrm{Ca}$ additional $2 \mathrm{~s}$ and $1 \mathrm{~d}$ orbitals are added. For open-shell nuclei like ${ }^{12} \mathrm{C}$ a fully antisymmetrized $\Phi_{\mathrm{A}}$ is much more complicated.

The central $f_{c}(r)$ and noncentral $u_{p}(r)$ pair correlation functions reflect the influence of the two-body potential at short distances, while satisfying asymptotic boundary conditions of cluster separability. Reasonable functions are generated by minimizing the two-body cluster energy of a somewhat modified $\mathrm{NN}$ interaction $(\bar{v}-\lambda)$, which contains a number of variational parameters. This leads to a set of eight coupled differential equations for the first eight operators [9]. The $f_{c}$ and $u_{6}=u_{t \tau}$ are shown in Figure 3 for several nuclei. Here $f_{c}(r)$ is small at short distances, to reduce the contribution of the repulsive core of the NN potential, and peaks at an intermediate distance corresponding to the maximum attraction of the NN potential. For light nuclei $f_{c}$ falls off at larger distances to keep the system confined. For closed shell nuclei confinement is provided by the one-body correlations and $f_{c}$ goes to a constant value at large distances. The noncentral $u_{p}(r)$ are all relatively small; the most important is the long- 
range tensor-isospin part $u_{t \tau}$, which is induced mainly by the one-pion-exchange part of the potential.

In calculations of five- and six-body nuclei the first four nucleons fill the $1 \mathrm{~s}$ shell and have no single-particle radial function, while the fifth and sixth nucleons go into the $1 \mathrm{p}$ shell. We allow for the possibility of different central correlations $f_{s s}, f_{p p}$, and $f_{s p}$ for pairs in the s-shell, pairs in the $\mathrm{p}$-shell, and mixed pairs, respectively. For five nucleons,

$\left|\Psi_{J}\right\rangle=A\left\{\prod_{1 \leq i<j \leq 4} f_{s s}\left(r_{i j}\right) \prod_{1 \leq k \leq 4} f_{s p}\left(\eta_{k 5}\right)\left|\Phi_{\alpha}(0000) \times \Phi_{p}\left(J M T T_{3}\right)\right\rangle\right\}$

$\left|\Phi_{\mathrm{p}}\left(\mathrm{JMTT}_{3}\right)\right\rangle=\phi_{\mathrm{p}}\left(\mathrm{R}_{5 \alpha}\right)\left[\mathrm{Y}_{1}^{\mathrm{m}_{1}}\left(\Omega_{5 \alpha}\right) \times \chi_{5}\left(\frac{1}{2} \mathrm{~m}_{\mathrm{S}}\right)\right]_{\mathrm{M}} v_{5}\left(\frac{1}{2} \mathrm{~T}_{3}\right)$

There is an explicit antisymmetrization over the five particles, and $\phi_{\mathrm{p}}$ is a single-particle correlation with $R_{i \alpha}=I_{i}-R_{\alpha}^{c m}$. The five-body nuclei ${ }^{5} \mathrm{He}$ and $5_{\mathrm{Li}}$ are not stable against breakup into an $\alpha$ and a nucleon, but we can study the scattering in both $J=3 / 2$ and $J=1 / 2$ states, using an R-matrix approach to minimize the energy inside a region with boundary conditions that correspond to an asymptotic scattering state [16. The difference in energy between these states is a measure of the spin-orbit splitting in nuclei, which in turn is the key to the nuclear shell model.

The six-body nuclei of interest are ${ }^{6} \mathrm{He}$ and ${ }^{6} \mathrm{Li}$ with $\mathrm{JMTT}_{3}=(001-1)$ and $(1100)$. They are weakly bound and easily broken into an $\alpha$ and a two-body cluster; ${ }^{6} \mathrm{Li}$ ( ${ }^{6} \mathrm{He}$ ) is only 1.5 $\mathrm{MeV}(1 \mathrm{MeV})$ more bound than a separated $\alpha$ and deuteron ( ${ }^{S_{\mathrm{O}}} \mathrm{nn}$ pair). However breakup into a five- plus one-body system costs $\sim 6 \mathrm{MeV}$ because the five-body nuclei are unstable. This dual character can probably best be modelled by a linear combination of shell-model and $\alpha$-cluster correlations:

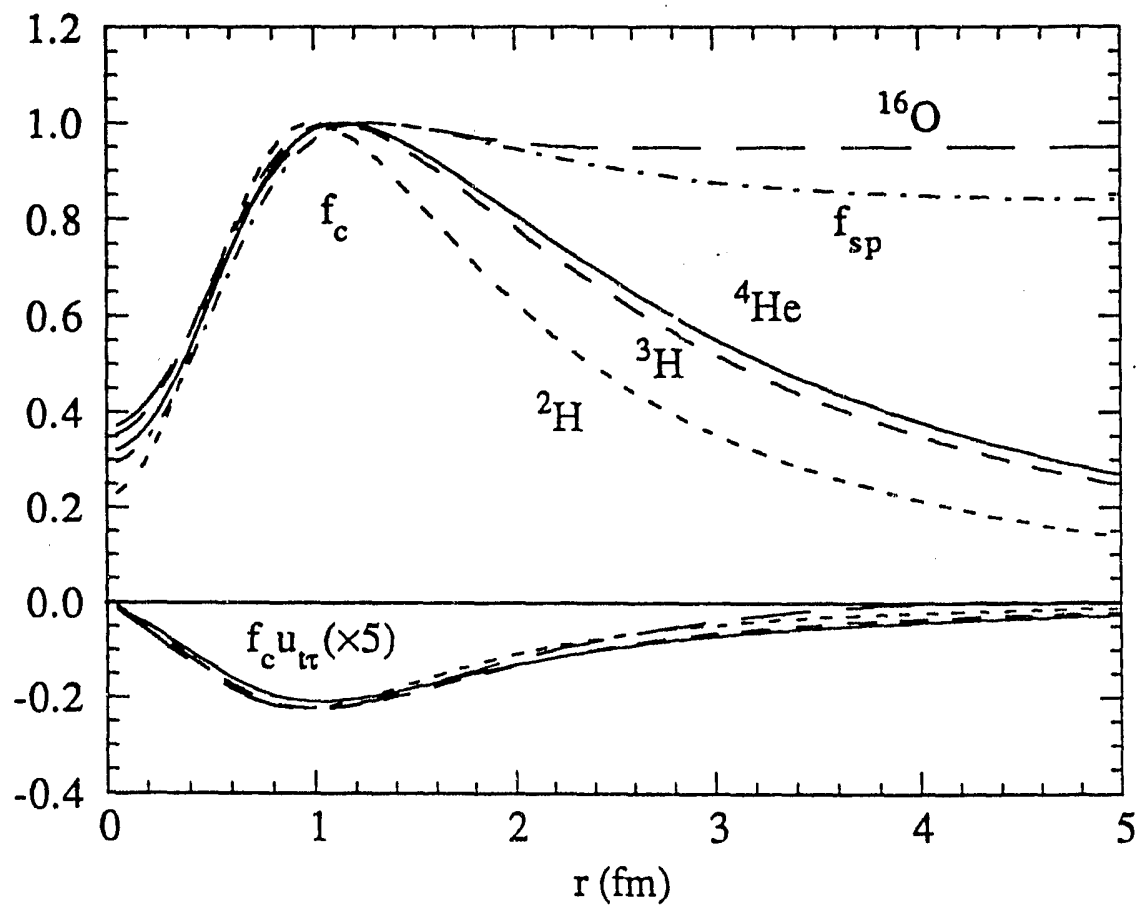

Figure 3. Correlation functions. 
$\left.|\Psi \mathrm{J}\rangle=A\left\{\prod_{1 \leq i<j \leq 4} f_{s s}\left(r_{i j}\right) \prod_{1 \leq k \leq 4} f_{s p}\left(r_{k 5}\right) f_{s p}\left(r_{k 6}\right) f_{p p}\left(r_{56}\right) l a \Phi_{s m}+L \Phi_{\alpha c}\right\rangle\right\}$

where the shell model wave function is:

$$
\begin{aligned}
\left|\Phi_{\mathrm{sm}}\right\rangle= & \Phi_{\alpha}(0000) \times \phi_{\mathrm{p}}\left(\mathrm{R}_{5 \alpha}\right) \phi_{\mathrm{p}}\left(\mathrm{R}_{6 \alpha}\right) \times \\
& \left\{\left[\mathrm{Y}_{1}^{\mathrm{m}_{1}}\left(\Omega_{5 \alpha}\right) \times \mathrm{Y}_{1}^{\mathrm{ml}}\left(\Omega_{6 \alpha}\right)\right]_{\mathrm{LM}_{\mathrm{L}}} \times\left[\chi_{5}\left(\frac{1}{2} \mathrm{~m}_{\mathrm{S}}\right) \times \chi_{6}\left(\frac{1}{2} \mathrm{~m}_{\mathrm{S}}^{\prime}\right)\right]_{\mathrm{SMS}_{\mathrm{S}}}\right\}_{\mathrm{JM}_{\mathrm{M}}}\left[\mathrm{v}_{5}\left(\frac{1}{2} \mathrm{t}_{3}\right) \times v_{6}\left(\frac{1}{2} \mathrm{t}_{3}\right)\right]_{\mathrm{TT}_{3}},
\end{aligned}
$$

while the $\alpha$-cluster correlation has s- and d-wave components induced by a tensor-like operator:

$$
\begin{aligned}
\left|\Phi_{\alpha c}\right\rangle= & \Phi_{\alpha}(0000) \times \\
& \left\{\left(\mathrm{u}\left(\mathrm{R}_{\alpha c}\right)+w\left(R_{\alpha c}\right)\left[3\left(\sigma_{5} \cdot \hat{R}_{\alpha c}\right)\left(\sigma_{6} \cdot \hat{R}_{\alpha c}\right)-\left(\sigma_{5} \cdot \sigma_{6}\right)\right]\right) \chi_{c}\left(\mathrm{SM}_{s}\right)\right\}_{J M} v_{c}\left(T_{3}\right) .
\end{aligned}
$$

Here $R_{\alpha c}$ is the separation between the $\alpha$ and two-body cluster centers of mass. The $f_{s s}(r) \approx$ $f_{c}\left({ }^{4} H e\right), f_{p p}(r) \approx f_{c}(2 H)$, and $f_{s p}(r)$ is shown in Figure 3 .

\section{CALCUlations OF FEW-BODY NUCLEI}

The method used to evaluate the energy expectation value varies with the size of the system under consideration. For small systems, $A \leq 8$, essentially exact integration is possible using Monte Carlo sampling [17]. For larger nuclei, $8 \leq A \leq 40$, a cluster expansion is required [11], with Monte Carlo techniques used to evaluate the terms in the expansion. For nuclear matter a cluster expansion is also used, but so far integral equation techniques have been used to sum. terms [12-14].

We represent $\Psi_{\mathrm{v}}(\mathbf{R})$ as a vector in spin-isospin space, with $\mathrm{N}_{\mathrm{S}} \times \mathrm{N}_{\mathrm{T}}$ complex numbers, where $N_{S}$ and $N_{T}$ are the number of spin and isospin basis states; $N_{x}=2 A$ in the basis of definite third components of spin or isospin. In the case of isospin, charge conservation can be used to reduce NT to $\mathrm{A} ! /[\mathrm{Z} !(\mathrm{A}-\mathrm{Z}) !]$, but a further reduction is possible by choosing states of definite $\mathrm{T}$ and $\mathrm{T}_{\mathrm{z}}$. In this latter basis, the vector lengths for ${ }^{3} \mathrm{H},{ }^{4} \mathrm{He},{ }^{5} \mathrm{He}$, and $6 \mathrm{Li}$ are 16,32 , 160 , and 320, respectively. The Metropolis Monte Carlo algorithm is used to perform the 3Adimensional integrals. Sampling is done both on the set of positions, $R=\left(\overrightarrow{\mathrm{r}}_{1}, \ldots \overrightarrow{\mathrm{r}}_{\mathrm{A}}\right)$, and the order of operators in the symmetrized product of eq.(8). For few-body nuclei a complete sum over all the spin-isospin variables is made. The computational effort required for this complete sum increases rapidly with $A$. The time required to calculate the binding energy with a reasonable statistical error for one trial function grows from about 3 minutes for ${ }^{3} \mathrm{H}$ to nearly 2 hours for ${ }^{6} \mathrm{Li}$ on a Cray-YMP. Thus direct integration will probably be limited to $\mathrm{A} \leq 8$ nuclei.

The results of variational calculations [9] for ground-state binding energies of ${ }^{3} \mathrm{H}$ and ${ }^{4} \mathrm{He}$ are shown in Table 1 along with preliminary results for ${ }^{5} \mathrm{He}$ and ${ }^{6} \mathrm{Li}$. For comparison we also show 34-channel Faddeev results [18] for ${ }^{3} \mathrm{H}$ and GFMC results [19] for ${ }^{3} \mathrm{H},{ }^{4} \mathrm{He}$ and ${ }^{5} \mathrm{He}$. There is also an older coupled-cluster result $[20]$ for ${ }^{4} \mathrm{He}$. The variational trial function gives upper-bound energies that are $3-4 \%$ above the exact results for ${ }^{3} \mathrm{H}$ and ${ }^{4} \mathrm{He}$. These and other calculations show that realistic $\mathrm{NN}$ potentials alone do not give enough binding for few-body nuclei, but it is possible to pick a supplemental NNN potential that will give the correct energy in exact calculations.

For ${ }^{5} \mathrm{He}$ the $\mathrm{J}=3 / 2$ and $\mathrm{J}=1 / 2$ scattering states have been calculated with a boundary condition that has a node in the $\alpha+n$ scattering wave function at $12.5 \mathrm{fm}$. The corresponding "experimental" energies, obtained from phase shift analyses, are 1.1 and $2.5 \mathrm{MeV}$ above the $\alpha$ binding energy, which give a spin-orbit splitting of $1.4 \mathrm{MeV}$. The variational results for the Argonne $\mathrm{v}_{14}+$ Urbana VIII model are 2.1 and $3.1 \mathrm{MeV}$ above the variational $\alpha$ binding energy 
Table 1

Binding and breakup energies (in $\mathrm{MeV}$ ) for few-body nuclei, with different Hamiltonians and calculational methods. Estimates of MC statistical errors are also given.

\begin{tabular}{|c|c|c|c|c|}
\hline nucleus & Hamiltonian & method & binding & breakup \\
\hline \multirow[t]{3}{*}{$3_{\mathrm{H}}$} & nature & experiment & 8.48 & \\
\hline & Reid v8 & $\begin{array}{l}\text { variational MC } \\
\text { GFMC } \\
\text { 34-ch. Faddeev }\end{array}$ & $\begin{array}{l}7.31 \pm 0.02 \\
7.54 \pm 0.10 \\
7.59\end{array}$ & \\
\hline & $\begin{array}{l}\text { Argonne v14 } \\
+ \text { Urbana VIII }\end{array}$ & $\begin{array}{l}\text { variational MC } \\
\text { 34-ch. Faddeev }\end{array}$ & $\begin{array}{l}8.21 \pm 0.02 \\
8.49\end{array}$ & \\
\hline \multirow[t]{3}{*}{${ }^{4} \mathrm{He}$} & nature & experiment & 28.3 & \\
\hline & $\begin{array}{l}\text { Reid } \\
\text { Reid v8 }\end{array}$ & $\begin{array}{l}\text { coupled cluster } \\
\text { variational MC } \\
\text { GFMC }\end{array}$ & $\begin{array}{l}24 . \\
23.6 \pm 0.1 \\
24.5 \pm 0.1\end{array}$ & \\
\hline & $\begin{array}{l}\text { Argonne v14 } \\
+ \text { Urbana VIII }\end{array}$ & $\begin{array}{l}\text { variational MC } \\
\text { GFMC }\end{array}$ & $\begin{array}{l}27.2 \pm 0.1 \\
28.3 \pm 0.2\end{array}$ & \\
\hline \multirow[t]{2}{*}{$5 \mathrm{He}\left(J=\frac{3}{2}\right)$} & nature & experiment & 27.2 & $-1.1(\alpha+n)$ \\
\hline & $\begin{array}{l}\text { Argonne v14 } \\
+ \text { Urbana VIII }\end{array}$ & $\begin{array}{l}\text { variational MC } \\
\text { GFMC }\end{array}$ & $\begin{array}{l}25.1 \pm 0.1 \\
26.8 \pm 0.2\end{array}$ & $\begin{array}{l}-2.1 \\
-1.5\end{array}$ \\
\hline \multirow[t]{2}{*}{$S_{H e}\left(\mathrm{~J}=\frac{1}{2}\right)$} & nature & experiment & 25.8 & $-2.5(\alpha+n)$ \\
\hline & $\begin{array}{l}\text { Argonne v14 } \\
\text { + Urbana VIII }\end{array}$ & $\begin{array}{l}\text { variational MC } \\
\text { GFMC }\end{array}$ & $\begin{array}{l}24.1 \pm 0.2 \\
25.6 \pm 0.2\end{array}$ & $\begin{array}{l}-3.1 \\
-2.7\end{array}$ \\
\hline \multirow[t]{2}{*}{${ }^{6} \mathrm{Li}$} & nature & experiment & 32.0 & $1.5(\alpha+d)$ \\
\hline & $\begin{array}{l}\text { Argonne v14 } \\
\text { + Urbana VIII }\end{array}$ & variational MC & $27.5 \pm 0.3$ & -1.9 \\
\hline
\end{tabular}

giving a splitting of $1 \mathrm{MeV}$. The GFMC results are 1.5 and $2.7 \mathrm{MeV}$ above the GFMC $\alpha$, which gives $1.2 \mathrm{MeV}$ or $85 \%$ of the "experimental" spin-orbit splitting with this Hamiltonian. The variational results required 25 hours of Cray-YMP time, including the parameter search, while the GFMC calculation required 200 hours, starting from a reasonable variational trial function.

For ${ }^{6} \mathrm{Li}$ only variational results are available at present, and they are unsatisfactory. The results shown here are for a trial function that gives the correct experimental charge radius, and while the energy is not terrible, it is nearly $2 \mathrm{MeV}$ above that of the corresponding separated $\alpha$ and deuteron. If the radius is unconstrained the trial function expands and the energy asymptotically approaches that of an $\alpha$ plus a deuteron with some residual Coulomb repulsion between them. Early results for ${ }^{6} \mathrm{He}$ show a similar problem. 


\section{CalCulations OF Light NUClei}

For larger nuclei we use a cluster expansion for the noncentral correlation operators $U_{i j}$, etc., to evaluate the energy expectation value. Consider the expansion for the NN potential:

$\left\langle\sum_{i<j} v_{i j}\right\rangle=\frac{\sum_{i<j} n_{i j}+\sum_{i<j<k} n_{i j k}+\ldots+n_{12 \ldots A}}{1+\sum_{i<j} d_{i j}+\sum_{i<j<k} d_{i j k}+\ldots+d_{12 \ldots A}}$

where the numerator and denominator cluster terms are given by:

$\mathrm{n}_{\mathrm{ij}}=\left\langle\Psi_{\mathrm{J}}\left|\left(1+U_{\mathrm{ij}}^{\dagger}\right) \mathrm{v}_{\mathrm{ij}}\left(1+U_{\mathrm{ij}}\right)\right| \Psi_{\mathrm{J}}\right\rangle$

$\mathrm{d}_{\mathrm{ij}}=\left\langle\Psi_{\mathrm{J}}\left|\left(1+\mathrm{U}_{\mathrm{ij}}^{\dagger}\right)\left(1+\mathrm{U}_{\mathrm{ij}}\right)\right| \Psi_{\mathrm{J}}\right\rangle-1$

$\mathrm{n}_{\mathrm{ijk}}=\left\langle\Psi_{\mathrm{J}}\left|\left[S \sum_{c y c}\left(1+\mathrm{U}_{\mathrm{ij}}^{\dagger}\right)\right]\left(1+\mathrm{U}_{\mathrm{ijk}}^{\dagger}\right) \sum_{c y c} \mathrm{v}_{\mathrm{ij}}\left(1+U_{\mathrm{ijk}}\right)\left[S \sum_{\mathrm{cyc}}\left(1+U_{\mathrm{ij}}\right)\right]\right| \Psi_{\mathrm{J}}\right\rangle-\sum_{c y c} \mathrm{n}_{\mathrm{ij}}$,

etc. The brackets denote a full 3A-dimensional integration, with the central correlations and antisymmetry of the Jastrow wave function treated completely. This may be regrouped as a linked cluster expansion:

$$
\begin{aligned}
& \left\langle\sum_{i<j} v_{i j}\right\rangle=\sum_{i<j} c_{i j}+\sum_{i<j<k} c_{i j k}+\ldots+c_{12 \ldots A}, \\
& c_{i j}=n_{i j} /\left(1+d_{i j}\right), \\
& c_{i j k}=\left[n_{i j k}-\sum_{c y c} c_{i j}\left(d_{j k}+d_{k i}+d_{i j k}\right)\right] /\left(1+\sum_{c y c} d_{i j}+d_{i j k}\right) .
\end{aligned}
$$

The expectation values of these individual cluster terms, $\mathrm{n}_{\mathrm{ij}}, \mathrm{d}_{\mathrm{ij}}$, etc., are evaluated using the same Monte Carlo techniques used for few-body nuclei. The spin-isospin algebra is only needed for the particles in the cluster that have noncentral correlation operators. However, the clusters are not in definite isospin states $T, T_{z}$ and only charge conservation can be used to reduce the size of the spin-isospin basis vector. The final 160 energy evaluation reported below required some 80 hours of Cray-2 time, after the trial function had been optimized.

The cluster expansion is not useful unless it converges rapidly. The convergence for $16 \mathrm{O}$ is reasonably good at the four-body cluster level, as shown in Table 2, where the contributions of the kinetic energy, NN and NNN potentials are shown at the one-, two-, three-, and four-body

Table 2

Convergence of cluster expansion for ground-state energy of 160 with Argonne $v_{14}+$ Urbana VII interaction. Energies are in $\mathrm{MeV} /$ nucleon.

\begin{tabular}{lcrrrrc}
\hline term & 1-body & 2-body & \multicolumn{1}{c}{ 3-body } & \multicolumn{1}{c}{ 4-body } & $\Sigma 1-4$ & $\Sigma 1-16$ \\
\hline $\mathrm{T}$ & $18.8 \pm 0.1$ & $17.2 \pm 0.1$ & $-1.7 \pm 0.1$ & $0.2 \pm 0.2$ & $34.5 \pm 0.3$ & 34.5 \\
$\mathrm{~V}_{\mathrm{ij}}$ & & $-46.8 \pm 0.2$ & $8.0 \pm 0.1$ & $-1.0 \pm 0.2$ & $-39.8 \pm 0.2$ & -39.7 \\
$\mathrm{~V}_{\mathrm{ijk}}$ & & & $-4.0 \pm 0.1$ & $2.2 \pm 0.1$ & $-1.8 \pm 0.1$ & -2.6 \\
$\mathrm{H}$ & $18.8 \pm 0.1$ & $-29.6 \pm 0.1$ & $2.3 \pm 0.1$ & $1.4 \pm 0.1$ & $-7.1 \pm 0.1$ & -7.8 \\
\hline
\end{tabular}


Table 3

Binding and breakup energies (in $\mathrm{MeV} /$ nucleon) for light nuclei with different Hamiltonians and calculational methods.

\begin{tabular}{llllc}
\hline nucleus & Hamiltonian & method & binding & breakup \\
\hline 160 & nature & experiment & 8.0 & $0.5(4 \alpha)$ \\
& Reid & coupled cluster & 5.0 & -1.0 \\
& Reid v6 & variational MC & $6.2 \pm 1.1$ & \\
& Argonne v14 & cluster MC & $7.8 \pm 0.3$ & 0.2 \\
& + Urbana VII & & & \\
& & & & \\
$40 \mathrm{Ca}$ & nature & experiment & 8.5 & \\
& Reid & coupled cluster & 6.0 & \\
& Argonne v14 & cluster MC & $8.6 \pm 1.5$ & \\
& + Urbana VII & & \\
\hline
\end{tabular}

cluster levels. The next to last column gives the sum of these terms through the four-body cluster level, while the last column gives an extrapolated estimate for the total energy through the sixteen-body cluster level. The kinetic energy converges rapidly, as does the NN potential contribution. The NNN potential has not converged at the four-body level, however, and there is a significant extrapolation in this term. Aln alternate cluster expansion, which may be more useful for open-shell nuclei like ${ }^{12} \mathrm{C}$, has been studied; in $16 \mathrm{O}$ it gives a similar extrapolated result for the $V_{i j k}$ expectation value.

Results for the ground-state binding energy per nucleon of $16 \mathrm{O}$ and ${ }^{40} \mathrm{Ca}$ are given in Table 3. A previous calculation of 160 using the coupled cluster method and the Reid potential has a problem similar to the ${ }^{6} \mathrm{Li}$ calculation discussed above; the nucleus is less bound per nucleon than ${ }^{4} \mathrm{He}$. A variational Monte Carlo calculation sampling all cluster sizes for the Reid v6 interaction also gaye too little binding, and had a large statistical error [21]. In the present calculation, with a realistic interaction including a three-nucleon potential, $16 \mathrm{O}$ is slightly more bound per nucleon than ${ }^{4} \mathrm{He}$, thus demonstrating stability for the first time. The result is also quite close to the experimental value, as is the preliminary result for ${ }^{40} \mathrm{Ca}$ with the same Hamiltonian. However, a variational search has not been made for the best trial function in ${ }^{40} \mathrm{Ca}$ and the present result has a large statistical uncertainty, as shown in the table.

Calculations are now in progress for a variety of other p-shell nuclei using the cluster Monte Carlo method, including ${ }^{8} \mathrm{He},{ }^{12} \mathrm{C}$, and ${ }^{15} \mathrm{~N}$. A first result for the spin-orbit splitting in ${ }^{15} \mathrm{~N}$ has been obtained by calculating the energy difference between states obtained by removing a single proton from either the $\mathrm{p}_{3 / 2}$ or $\mathrm{p}_{1 / 2}$ shell in the trial function for $16 \mathrm{O}$. The calculated spin-orbit splitting is $6.1 \pm 0.9 \mathrm{MeV}$, in reasonable agreement with the experimental value of $6.3 \mathrm{MeV}$. About $? / 3$ of the splitting comes from L-dependent terms in $v_{i j}$ or $\Psi_{V}$, and about $1 / 3$ comes from $V_{i j k}$ terms. This result is also in general agreement with earlier Brueckner G-matrix calculations that used the Reid $v_{i j}$ and Tucson-Melbourne $V_{i j k}[22]$.

\section{FORM FACTORS AND MOMENTUM DISTRIBUTIOHS}

Other properties which we have studied include the electromagnetic form factors and nucleon momentum distributions. The form factors have significant contributions from twobody charge and current operators attributable to meson exchange (MEC). These include both a "model-independent" (MII) part which is constrained by requiring current conservation with 
Table 4

Magnetic moments for $\mathrm{A}=3$ nuclei

\begin{tabular}{|c|c|c|c|c|}
\hline & \multicolumn{2}{|c|}{${ }^{3} \mathrm{H}$} & \multicolumn{2}{|c|}{${ }^{3} \mathrm{He}$} \\
\hline & variational & Faddeev & variacional & Faddeev \\
\hline IA & 2.592 & 2.588 & -1.782 & -1.776 \\
\hline $\mathrm{IA}+\mathrm{MI}$ & 2.988 & 2.968 & -2.144 & -2.122 \\
\hline $\mathrm{IA}+\mathrm{MI}+\mathrm{MD}$ & 3.031 & 3.010 & -2.175 & -2.152 \\
\hline experiment & \multicolumn{2}{|c|}{2.979} & \multicolumn{2}{|c|}{-2.127} \\
\hline
\end{tabular}

the chosen $v_{i j}$, and a "model-dependent" $(M D)$ part that includes currents associated with $p \pi \gamma$, $\omega \pi \gamma$, and $\Delta$-excitation mechanisms $[23,29]$. Results for the magnetic moments of ${ }^{3} \mathrm{H}$ and ${ }^{3} \mathrm{He}$ are given in Table 4 and for the charge form factor of ${ }^{4} \mathrm{He}$ in Figure 4 . These include impulse approximation (IA) calculations with and without MEC for both variational and exact calculations. The results show that the few-body variational trial functions are good for more than just the energy, and that the MEC are essential for quantitative agreement with data. The charge form factor for 160 is also shown in Figure 4; here the present variational wave function is not as satisfactory and the charge radius is too small.

The nucleon momentum distribution is given by the integral:

$\rho(k)=\int d R \sum_{i} \int d \vec{r}_{i}^{\prime} \Psi\left(R^{\prime}\right) \exp \left[-i \vec{k} \cdot\left(\vec{r}_{i}-\vec{r}_{i}^{\prime}\right)\right] \Psi(\mathbf{R})$,

where $\mathbf{R}^{\prime}=\left(\vec{r}_{1}, \ldots, \vec{r}_{i^{\prime}}, \ldots \vec{r}_{A}\right)$. The operator expectation values are calculated by direct

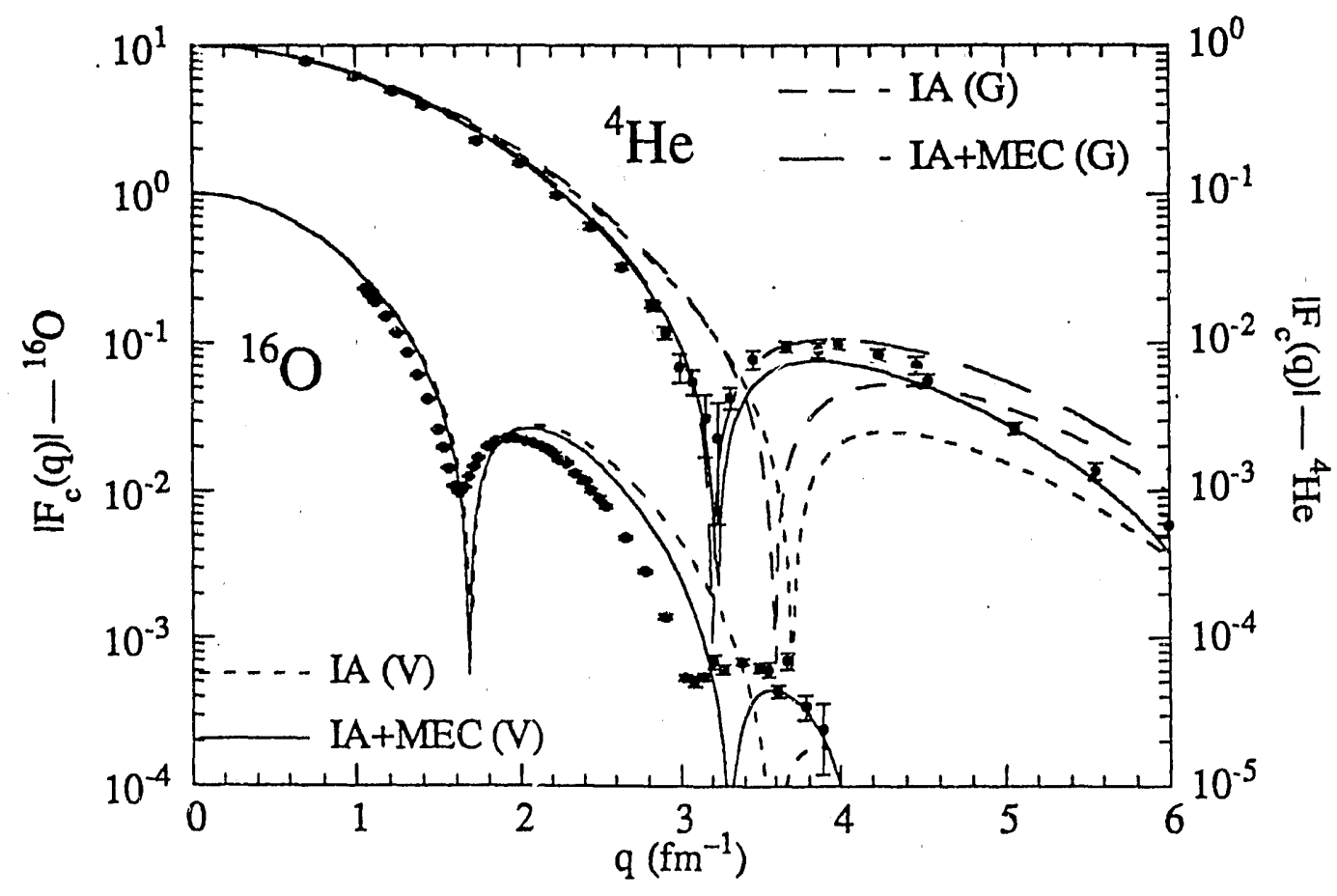

Figure 4. Charge form 
integration in the few-body nuclei, but a cluster expansion is again needed for larger systems. Results for ${ }^{4} \mathrm{He}$ and ${ }^{16} \mathrm{O}$ are shown in Figure 5 along with the exact deuteron and a correlated basis function calculation of nuclear matter [24]. All these systems show a remarkably similar high-momentum tail, which is indicative of the strong nuclear correlations; this tail may be useful in understanding phenomena such as low-energy pion and antiproton production.

\section{LOW-ENERGY ELECTROWEAK TRANSITIONS}

We have also begun to adapt the variational methods to the study of low-energy fewnucleon reactions. Faddeev methods have already been used with success in the study of three-nucleon reactions $[25,26]$, but variational methods are the best available for the study of four-nucleon reactions at present. Our initial studies have been of the electroweak capture reactions: ${ }^{2} \mathrm{H}(\mathrm{d}, \gamma){ }^{4} \mathrm{He}$ below $500 \mathrm{keV}$ c.m. energy, ${ }^{3} \mathrm{He}(\mathrm{n}, \gamma){ }^{4} \mathrm{He}$ with thermal neutrons, and ${ }^{3} \mathrm{He}\left(\mathrm{p}, \mathrm{e}^{+} v_{\mathrm{e}}\right)^{4} \mathrm{He}$ at solar energies [27-29]. The first reaction is discussed by Arriaga elsewhere in this conference; here we discuss the two capture reactions on ${ }^{3} \mathrm{He}$. There is good recent data for the thermal neutron capture, while the weak proton capture is of interest because its Hep neutrinos might be detectable in the next generation of solar neutrino experiments.

These two capture reactions have very small IA contributions due to a pseudo-orthogonality between the dominant S-wave parts of the nucleon- ${ }^{3} \mathrm{He}$ continuum state and ${ }^{4} \mathrm{He}$ ground state. MEC contributions are very important, and accurate evaluation of two-body current operators requires good correlated wave functions. In this case, the continuum wave functions were generated for the Argonne $v_{14}+$ Urbana VIII Hamiltonian with methods similar to those used for studying ${ }^{5} \mathrm{He}$. The scattering lengths obtained are $3.50 \pm 0.25 \mathrm{fm}$ for $\mathrm{n}+{ }^{3} \mathrm{He}$, and $10.1 \pm 0.5$ fru for $\mathrm{p}+{ }^{3} \mathrm{He}$ (the errors are Monte Carlo statistics), in excellent agreement with effectiverange parametrizations of low-energy data which give $3.52 \pm 0.25 \mathrm{fm}$ and $10.2 \pm 1.4 \mathrm{fm}$, respectively.

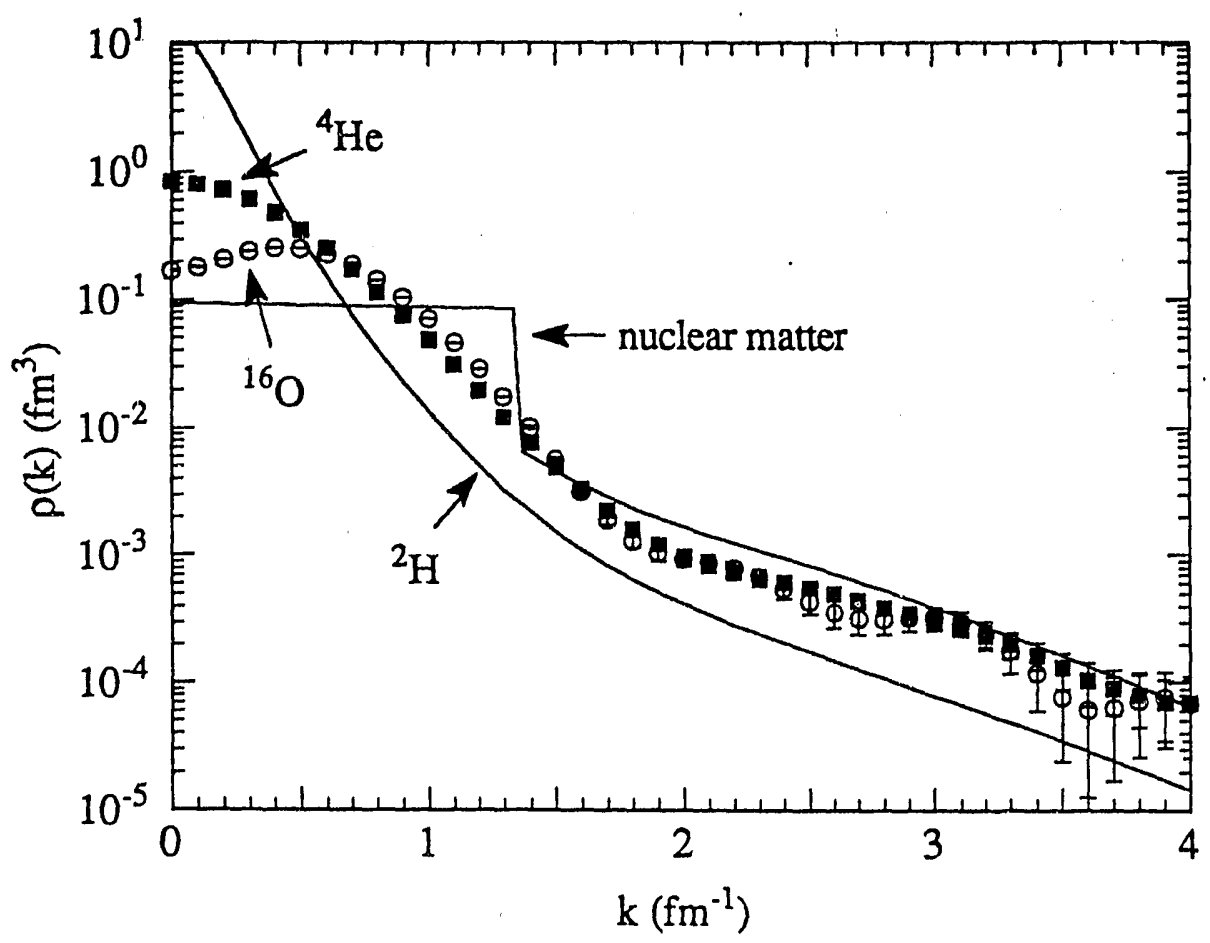

Figure 5. Nucleon momentum distributions. 
The IA cross section for thermal neutron capture on ${ }^{3} \mathrm{He}$ is only $6 \mu \mathrm{b}$, compared to the experimental value of $55 \mu \mathrm{b}$. The MEC contributions again include a MI part, and mesonic and intermediate $\Delta$-isobar MD parts. The $\mathrm{MI}$ matrix element is opposite in sign to the $1 \mathrm{~A}$, and much larger, raising the calculated cross section to $72 \mu \mathrm{b}$. In our first studies, the $\Delta$-isobar contributions were calculated with a standard first-order perturbation estimate, and raised the total calculated cross section to $112 \mu \mathrm{b}$ [28]. More recently, we have used explicit $\Delta$-isobar components in the wave functions, introduced by transition correlation operators:

$\Psi=\left[S \prod_{i<j}\left(1+U_{i j}^{T R}\right)\right] \Psi_{N}=\left[S \prod_{i<j}\left(1+U_{i j}^{N \Delta}+U_{i j}^{\Delta N}+U_{i j}^{\Delta \Delta}\right)\right] \Psi_{N}$

where $\Psi_{N}$ is the nucleons-only wave function [29]. The correlations $U_{i j}^{B B}$ are generated by an approximate fit to exact two-body calculations with the Argonne v28 potential [2], which has explicit $\Delta$-isobar degrees of freedom and is phase equivalent to the v14 potential. Contributions of $\Delta$-isobars to the electroweak current operators then appear at the one-body level, and oneand two- $\Delta$ components are evaluated. This approach should give more reliable results than first-order perturbation theory. The final themral neutron capture cross section is significantly reduced, to a value of $86 \mu \mathrm{b}$. This improved treatment of the $\triangle$-isobar MEC contributions is also included in the magnetic moment calculations of Table 4.

The astrophysical S-factor for the zero-energy proton weak capture on $3 \mathrm{He}$ has an IA value of $6.9 \times 10^{-23} \mathrm{MeV}-\mathrm{b}$. The MEC contributions are all MD, and in order to reduce uncertainty in their contributions, they are adjusted to reproduce the Gamow-Teller matrix element in ${ }^{3} \mathrm{H}$ $\beta$-decay. Again, the MEC matix element is opposite in sign to the IA, but not so big, resulting in a reduction of the S-factor to $1.4 \times 10^{-23} \mathrm{MeV}-\mathrm{b}$. Both the thermal neutron radiative capture and the zero-energy proton weak capture are very sensitive to the nucleon +3 He scattering length, and the results can vary by $\pm 20-30 \%$ as the scattering length is changed within the theoretical limits (which are smaller than the experimental errors).

\section{CONCLUSIONS}

Good progress has been made in the microscopic study of nuclear structure for few-body and light nuclei in recent years. Current work is now concentrating on the ground states of intermediate size systems, $4<A \leq 40$, where the number of nucleons is large enough to make calculations difficult, yet small enough that the finite nature of the system is an important aspect of the physics. Studies will also continue on a variety of low-energy few-body reactions.

The difficulty in explaining the stability of light nuclei may be due to inadequacies in the variational ansatz, or it may be due to the crude parametrization of the NNN potential. Obtaining a consistent description of nuclear systems with realistic interactions remains a challenging problem. Continued progress will require advances in the many-body Hamiltonian, the many-body theory, and the available computational resources.

\section{ACKNOWLEDGMENTS}

The work described here has been carried out in collaboration with A. Arriaga, J. Carlson, V. R. Pandharipande, S. C. Pieper, D. O. Riska, and R. Schiavilla. Computations have been made at the National Energy Research Supercomputer Center in Livermore, California, and at the National Center for Supercomputer Applications in Urbana, Ilinois. This work is partially supported by the U.S. Department of Energy, Nuclear Physics Division, under contract W-31109-ENG-38. 


\section{REFERENCES}

[1] R. V. Reid, Ann. Phys. (N.Y.) 50, 411 (1968).

[2] R. B. Wiringa, R. A. Smith, and T. L. Ainswortl, Phys. Rev. C 29, 1207 (1984).

[3] R. Machleidt, K. Holinde, and Ch. Elster, Phys. Rep. 149, 1 (1987).

[4] N. M. Nagles, T. A. Rijken, and J. J. de Swart, Phys. Rev. D 17, 768 (1978).

[5] M. Lacombe, B. Loiseau, J. M. Richard, R. Vinh Mau, J. Côte, P. Pirés, and R. de Tourreil $_{\text {i }}$ Phys. Rev. C 21, 861 (1980).

[6] J. Fujita and H. Miyazawa, Prog. Theor. Phys. 17, 360 (1957).

[7] J. Carlson, V. R. Pandharipande, and R. B. Wiringa, Nucl. Phys. A401, 59 (1983).

[8] R. Schiavilla, V. R. Pandharipande, and R. B. Wiringa, Nucl. Phys. A449, 219 (1986).

[9] R. B. Wiringa, Phys. Rev. 43, 1585 (1991).

[10] S. A. Coon, M. D. Scadron, P. C. McNamee, B. R. Barrett, D. W. E. Blatt, and B. H. J. McKellar., Nurl. Phys. A317, 242 (1979).

[11] S. C. Pieper, R. B. Wiringa, and V. R. Pandharipande, Phys. Rev. Lett. 64, 364 (1990).

[12] V. R. Pandharipande and R. B. Wirin a, Rev. Mod. Phys. 51, 821 (1979).

[13] I. E. Lagaris and V. R. Pandharipande, Nucl. Phys. A359, 349 (1981).

[14] R. B. Wiringa, V. Fiks, and A. Fabrocini, Phys. Rev. C 38, 1010 (1988).

[15] J. Carlson, Fhys. Rev. C 38, 1879 (1988); Nucl. Phys. A508, 141c (1990).

[16] J. Carlson, K. E. Schmidt, and M. H. Kalos, Phys. Rev. C 36, 27 (1987).

[17] J. Carlson and R. B. Wiringa, in Computational Nuclear Physics 1, edited by K. Langanke, J. A. Maruhn, and S. E. Koonin (Springer Verlag, Berlin, 1991).

[18] C. R. Chen, G. L. Payne, J. L. Friar, and B. F. Gibson, Phys. Rev. C 33, 1740 (1986); private communication.

[19] J. Carison, private communication

[20] H. Kümmel, K. H. Lührmann, and J. G. Zabolitzky, Phys. Rep. 36C, 1 (1978).

[21] J. Carlson and M. H. Kalos, Phys. Rev. C 32, 2105 (1985).

[22] K. Ando and H. Bando, Prog. Theor. Phys. 66, 227 (1981).

[23] R. Schiavilla, V. R. Pandharipande, and D. O. Riska, Phys. Rev. C 40, 2294 (1989); Phys. Rev. C 41, 309 (1990).

[24] S. Fantoni and V. R. Pandharipande, Nucl. Phys. A427, 473 (1984).

[25] W. Glöckle, H. Witala, and Th. Cornelius, Nucl. Phys. A508, 115c (1990).

[26] C. R. Chen, G. L. Payne, J. L. Friar, and B. F. Gibson, Phys. Rev. C 44, 50 (1991).

[27] A. Arriaga, V. R. Pandharipande, and R. Schiavilla, Phys. Rev. C 43, 983 (1991).

[28] J. Carlson, D. O. Riska, R. Schiavilla, and R. B. Wiringa, Phys. Rev. C 42, 830 (1990); Phys. Rev. C44, 619 (1991).

[29] R. Schiavilla, R. B. Wiringa, V. R. Pandharipande, and J. Carlson, Argonne National Laboratory Physics Division preprint PHY-6898-TH-91 (1991). 

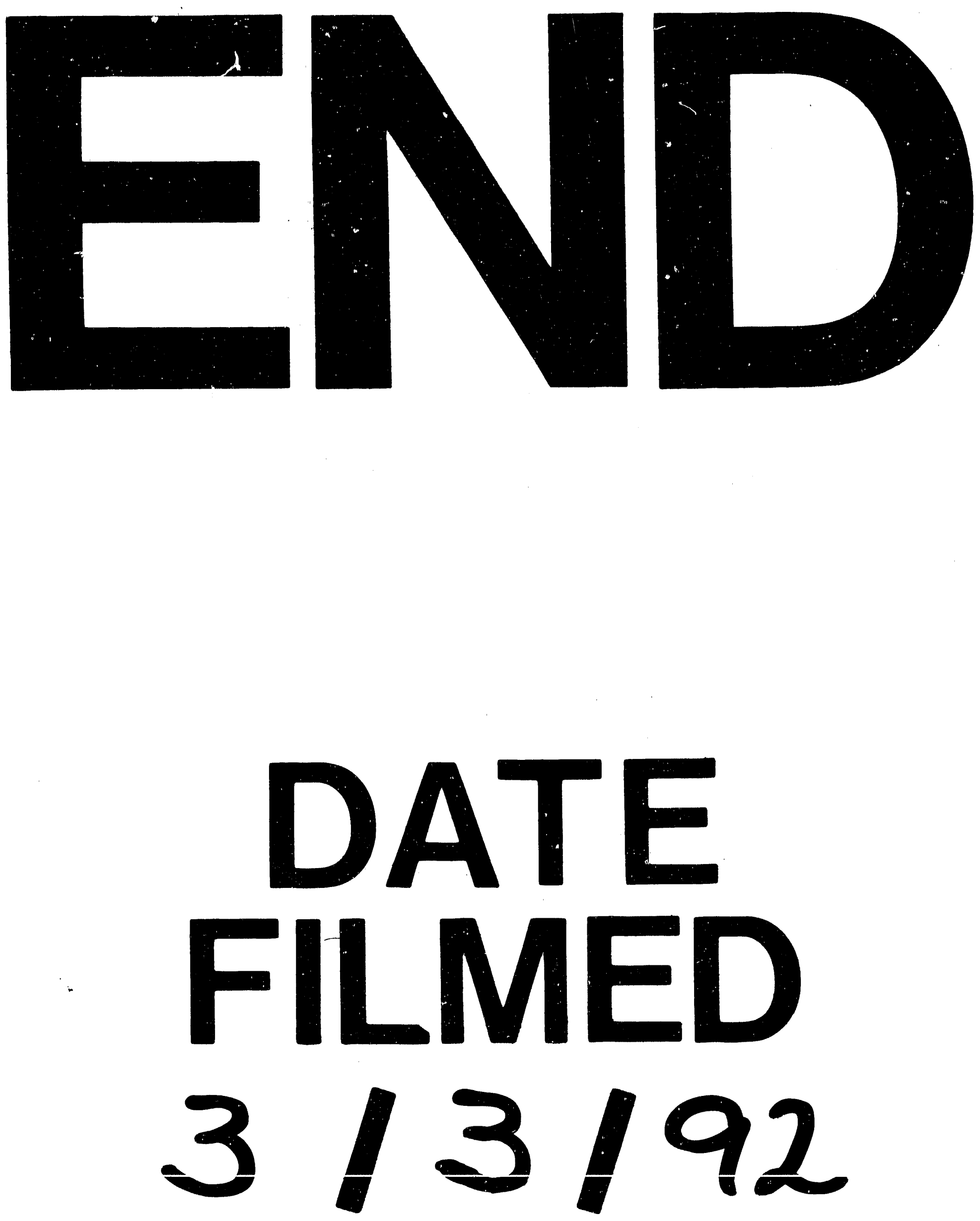
$m$ 